\title{
Optimal Spectrum Utilization and Flow Controlling In Heterogeneous Network with Reconfigurable Devices
}

\author{
Syed Shakeel Hashmi, Syed Abdul Sattar, and K. Soundararajan
}

\begin{abstract}
Fairness provisioning in heterogeneous networks is a prime issue for high-rate data flow, wherein the inter-connectivity property among different communication devices provides higher throughput. In Hetnet, optimal resource utilization is required for efficient resource usage. Proper resource allocation in such a network led to higher data flow performance for real-time applications. In view of optimal resource allocation, a resource utilization approach for a reconfigurable cognitive device with spectrum sensing capability is proposed in this paper. The allocation of the data flow rate at device level is proposed for optimization of network fairness in a heterogeneous network. A dynamic approach of rate-inference optimization is proposed to provide fairness in dynamic data traffic conditions. The simulation results validate the improvement in offered quality in comparison to multi-attribute optimization.
\end{abstract}

Keywords - rate-inference optimization, heterogeneous network, flow control, spectrum utilization

\section{INTRODUCTION}

$\mathbf{W}$ ITH regard to emerging trends in wireless communication, heterogeneous networks represent an evolving area. A heterogeneous network (HetNet) is acquired as a cluster of distinct subnetworks, which are grouped together to maintain long-range communication with different wireless communication architectures. The integrated mode approach of communication has resulted in efficient communication, with higher throughput performance for wireless communication. The architecture is in development to suite the upcoming requirements of demanded services for next-generation wireless communication. Such networks are evolving towards a new mode of communication; various wireless-enabled networks are integrated together to support long-range communication, and the optimality issues with respect to resource utilization and usage are under investigation. Referring to the changed format of wireless networks integrated together, the resources are to be properly controlled to achieve the objective of fairness among the distributed networks with user compatibility. For optimal resource allocation, various methods have been developed in the past, e.g., spectrum allocation, routing optimization, and power efficiency.

For the development of optimal resource allocation in heterogeneous networks, an approach for the optimal allocation of resources in terms of spectrum efficiency, fairness and battery power during communication is outlined in [1]. A multi-

S. S. Hashmi is with the ECE Department, Icfai Tech School, IFHE University and Research Scholar at JNTU Anantapur, India (e-mail: hashmi@ifheindia.org)

S. A. Sattar is with ECE Department, NSAKCET, Hyderabad, India (e-mail: syedabdulsattar1965@gmail.com).

K. Soundararaj is with ECE Department, NSACET, Hyderabad, India (email: soundararajan.tkr@gmail.com). attribute scheduling algorithm approach is proposed, where these parameters are used for resource scheduling. This multiattribute approach is significant because it controls the allocated data rate based on an optimized utility function in the HetNet. This optimization is proved wrt. optimal rate allocation over maximization of the spectrum efficiency and fairness factor. In order to be fair to the user, an interference alignment (IA) scheme for resource allocation was presented in [2]. A spectral coexistence mechanism to mitigate the interference in heterogeneous networks was presented. The resource allocation in terms of optimal interference management was advised. In [3], the optimality issue of resource allocation with respect to traffic density was outlined. The offered capacity for transmission was governed by the solution of a continuous linear programming (LP) approach, where a solution is derived by a scheduler unit based on the number of mobiles in the network. A weighted distribution scheme for all the available weights in the network is presented in [4]. The approach of resource allocation based on an existing wireless network for user preference and mobile terminal power is presented. A cost optimization approach was derived for different networks of macro-, pico-, femto- and relay-based communication approaches towards service uniformity in these networks. In [5], a topology-governed inference management for enhancing the performance of a heterogeneous network is outlined. To obtain the objective of optimal resource allocation in heterogeneous networks, an adaptive interference coordination in inter-cellular interference for smart resource allocation is presented. An intracellular fairness objective was suggested. In [6],[7], a new dynamic scheduling over a heterogeneous network, following packet scheduling, was presented. A mediaindependent handover concept has been realized to offer efficient transmission of multimedia services using a heterogeneous network. For provisioning of the spectral efficiency in a heterogeneous network, a cell range expansion (CRE) problem was addressed in [8],9] by setting a positive bias factor in the short-range communication network. The objective was derived as the average area weighted load per cell, defined as the weighted spectrum efficiency and energy efficiency approach. With a similar objective for the spectral efficiency in [10],11], the spectrum efficiency (SE) and energy efficiency (EE) derived over a realistic network were presented. An operation relation and quality governance for efficient coding was suggested. In [12], SE and EE objectives were analysed using a biased inter- and intra-radio access technologies (RAT) offloading technique. The analysis yields a multi-objective optimization problem, maximizing the SE and EE subjected to the bounding quality of services. Most of the approaches are developed for resource optimization, and the offering quality of services is an observable parameter. To offer 
QoS in heterogeneous networks, a quality objective with regard to an interference constraint across the cell coverage is presented in [13]. The QoS parameters are defined as a function of link bandwidth, node buffer and queuing service and are employed at the router level in a heterogeneous network. In [14], an end-to-end QoS framework for heterogeneous networks is focused on. The objective of QoS provisioning was made over multiple autonomous systems. In [15],16], a routing algorithm based on proactive coding was suggested, and a QoSdriven control selection was outlined. A general backward utility formation was introduced to observe the dynamic QoS variation in the network. With respect to the resource variation issue and governing of QoS, multi-hop communication with regard to homogeneous and heterogeneous networks was presented in [17]. The variant parameter of a micro-site with regard to a traffic load condition was analysed. In [18],19], IPoriented QoS signalling as a function of end-to-end QoS offering and seamless mobility for joint resource management was presented. The approach of node automaticity and quality monitoring was presented with mobility concerns for QoS offering. The method derived from supporting various services in different architectures of heterogeneous networks. In providing QoS, spectrum allocation based on the device level is presented in [20]. An energy-efficient cooperative spectrum sensing strategy for immune noise uncertainty was presented. Coarse and fine sensing schemes are employed to achieve energy efficiency in HetNets. A relation property of noise immunity over power consumption was derived. To offer resource utilization via sharing, a device-to-device-level coding is presented in [21] based on a spectrum partitioning approach, and an improved spectrum efficiency for D2D and cellular networks was derived. Under quality-of-service governance, the spectrum is divided into several subfrequencies, and each band is allocated a D2D pair for transmission. Optimality was observed for maximization of D2D transmission power. The approach was developed under the constraint of offering QoS to the cellular transmission conformation. In [22], a devicelevel coding using cognitive radio units (CRNs) is presented. An active resource allocation problem for heterogeneous networks under imperfect channel conditions was presented The optimality issue was resolved by applying an optimal power allocation problem under a known channel condition. In the suggested approach under network abstraction, the node operating as a router was controlled via different approaches to achieve the objective of higher performance in a heterogeneous network. In [23], a bandwidth buffering mechanism addressing the allocation issue and different queuing services to provide end-to-end QoS provision in heterogeneous networks was suggested. isA self-clocked fair queuing mechanism for providing QoS governance was presented, and the issues of the end-to-end requirement and user connectivity were addressed. While the provision of optimal quality of service and fairness was solved in different aspects, multi-objective control for HetNets is still to be developed to achieve optimal equilibrium in resource utilization with offered QoS and network operation.

In this paper, a new approach for quality-governed data flow operation is outlined to achieve efficient operational performance in heterogeneous networks. An integration approach for device performance and network control is proposed to reach the target of higher performance in a heterogeneous network. The remainder of this paper is organized as follows. Section II outlines the system model used for the developed approach. The conventional model of the multi-attribute optimization approach, based on spectral efficiency and fairness as outlined in [1], is presented in section III. Section IV outlines the proposed spectrum sense optimization approach and rate flow control for heterogeneous networks. Section V illustrates the experimental results derived from the proposed approach in comparison to conventional modelling.

\section{SYSTEM MODEL}

For the communication of data, a cluster of subnetworks capable of wireless transmission is used. An instance of the network model following the heterogeneous network representation is shown in Fig. 1.

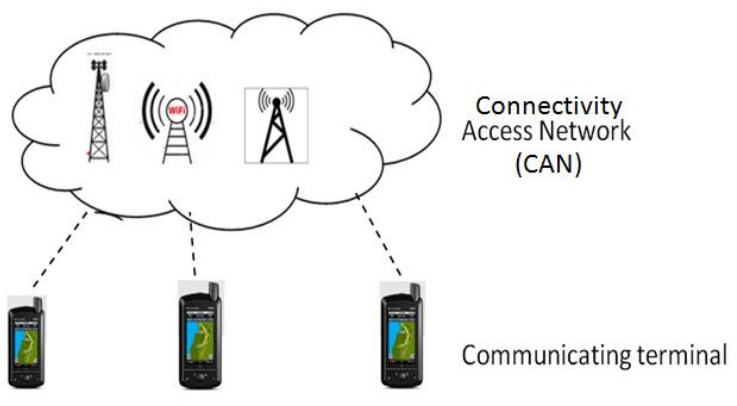

Fig. 1. Heterogeneous Architecture

The network is defined with a set of communication terminals, defined as cognitive user units (CuEs), where each device has the property of cognitive operation. In this network, the communication terminals are linked with access points $\left(\mathrm{AP}_{\mathrm{i}}\right)$, where each of these access points is set with different communication standards. The access network is defined with a Wi-Fi terminal, a cellular terminal and a WiMAX link point. In the process of communication, a communication terminal requests access. Based on the available access terminal, an access link is selected to offer maximum resources. The selected access terminal is then linked with the communication terminal via a GRCU (global resource control unit). To perform the resource allocation, the communication terminal registers the selected access terminal to the GRCU, and then a handshake process is carried out between the GRCU and the communication terminal, confirming the allocation. Once the allocation is confirmed, communication is established from the communication terminal to the destination terminal via the selected access point, controlled by the GRCU. The flow control of the heterogeneous network is illustrated in Fig. 2.

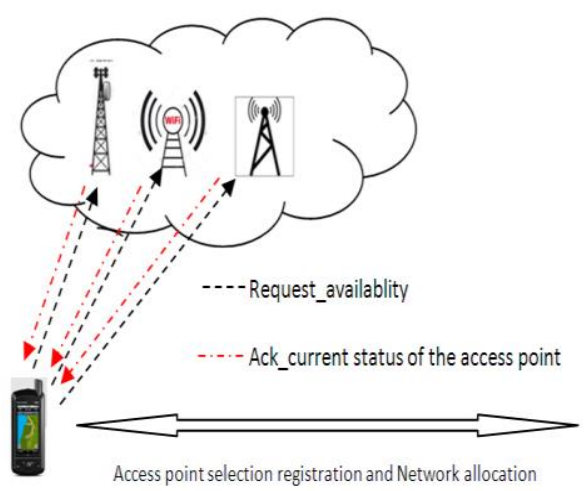

Fig. 2. Control flow diagram of the considered Heterogeneous Network 
During the process of communication, a terminal access was granted by the GRCU, registering the sensed access point with the highest available resource. The resource allocations take place in the GRCU unit, and an optimal data rate is allocated based on the current state of the registered access units. For resource allocation, the GRCU unit operates with a multiattribute optimization approach as outlined in [1]. The conventional approach of multi-attribute optimization is outlined in the next section.

\section{Multi-Attribute OPTIMIZATION APPROACH [1]}

To provide efficient coding for resource allocation in heterogeneous networks considering Wi-Fi, 4G (LTE/WiMAX), and 3G (HSPA/EVDO) architectures, a multiattribute optimization approach is suggested in [1]. In the process of the multi-attribute optimization approach, 3 factors were considered: 1) Spectral efficiency, 2) Fairness and 3) Battery life. Spectral efficiency is calculated as the utility function of the allocated rate over the total spectrum $k$ available at time $\mathrm{t}$ for $\mathrm{u}$ users belonging to a set of users $\mathrm{U}$, i.e.,

$$
\gamma^{t}=\frac{\sum_{u \in U} r_{u}^{t}}{k}
$$

where the allocated rate $r_{u}^{t}$ is defined by

$$
r_{u}^{t}=\sum_{a \in A} x_{u a}^{t} * r_{u a}^{t}
$$

where $\mathrm{A}$ is the set of $\mathrm{BS} / \mathrm{APs}$ registered and $a \in A$ is the radio offered to $\mathrm{A}$ at time $\mathrm{t}$.

The optimal spectral allocation is obtained by optimizing the allocation rate, defined by the maximum sum rate (MSR) optimization problem. However, the MSR has a limitation for the global user resource allocation problem, which yields an unfairness in spectrum allocation. To optimize the spectrum allocation, a utility factor $\gamma_{u t i l}^{t}$ is used, which is defined by [1] as

$$
\gamma_{u t i l}^{t}=\frac{\gamma^{t}-\gamma_{\min }^{t}}{\gamma_{\max }^{t}-\gamma_{\min }^{t}}
$$

where $\gamma_{\text {max }}^{t}$ is the maximum achievable spectral efficiency utility function and $\gamma_{\min }^{t}$ is the minimum spectral utility function. When the network achieves $\gamma_{\max }^{t}$ efficiency, the utility function is given a value of 1 , while the value is 0 when the achieved spectral efficiency is $\gamma_{\text {min }}^{t}$. To provide a fair platform, the allocated rate is controlled by a fairness utility function $\theta_{u t i l}^{t}$. This utility function $\theta_{u t i l}^{t}$ is defined by [1] as,

$$
\theta_{u t i l}^{t}=1-\frac{\left|B U^{t}\right|}{|U|}
$$

The fairness utility function is defined as a function of user blockage, where it takes a value of 1 when the number of blocked users (BUs) $=0$ and takes a value of 0 when the total blockage is maximal. For long-term fairness, the utility function is defined by [1] as

$$
\phi_{u t i l}=\frac{\left(\sum_{u \in U} \sum_{t} r_{u}^{t}\right)^{2}}{|U| * \sum_{u \in U}\left(\sum_{t} r_{u}^{t}\right)^{2}}
$$

where the long-term fairness is derived by the aggregated observation of long-term small rate allocations. As a third attribute, battery life is considered for optimal resource allocation. The utility factor under this objective is defined by [1] as

$$
\omega_{u t i l}^{t}=1-\frac{\sum_{u \in U} \omega_{u}^{t}-\omega_{\min }^{t}}{\omega_{\max }^{t}-\omega_{\min }^{t}}
$$

where $\omega_{u}^{t}$ is the utility function for the battery life of each user. These multiple attributes are controlled, to obtain an efficient coding for spectral efficiency, fairness and long life of nodes in a heterogeneous network. However, the concerns of device efficiency and service quality have not been focused on. The optimization is derived based on the utility factors of these attributes, and the control is made wrt. the allocated rate at time t. To achieve higher efficiency in resource utilization with a quality concern in such Heterogeneous Networks, a qualitygoverned approach with device reconfiguration is proposed.

\section{PRoposed OPtIMIZATION ATtRIBUTE}

\section{A. Spectrum sense optimization}

In the present approach, during the initialization process, the cognitive user equipment $(\mathrm{CuE})$ performs a search operation in Connectivity Access Networks (CANs) based on the order of network preference. In this approach, the order of preference is set as Wi-Fi, WiMAX and Cellular networks. For each of these networks, a utility factor is calculated, and the network offering the higher fairness factor is chosen. The selected CAN is registered with the GRC, and communication is then formed over the registered CAN. In the process of communication, the GRC allocates the spectrum for communication under the limitation of $\gamma_{u t i l} \in[0,1]$. The rate allocation is based on the demanded spectrum to the GRC, where the GRC performs multi-attribute resource allocation to allocate resources to the requested unit. However, with the communication process, the spectrum is dynamically engaged and released based on the demanded service. The vacant spectrum can be utilised for current active users towards extra spectrum allocation to improve service quality. As the devices are cognitive in nature, the cognitive property of resource allocation can be used for these spectrum allocations. The initial spectrum allocation for user $\mathrm{u} € \mathrm{U}$ is given by the utility value $\gamma_{U}^{t}$. On the release of a primary allocation rate $r_{i}^{t}$, the available spectrum is defined by

$$
\gamma_{a v 1}^{t}=\sum_{u \in U} \gamma_{u}^{t}-r_{i}^{t}
$$

This available spectrum is called the secondary spectrum and can be utilised by the active primary users $\left(p_{u}\right)$. On the request for this secondary spectrum, the GRCU computes the spectral interference energy, $E$, for n active user as defined by [20] as

$$
E=\sum_{i=1}^{n}\left(\frac{y_{i}}{\sigma}\right)^{P}
$$

where $y_{i}$ is the received information and $\sigma$ is the observed channel variance. Parameter ' $P$ ' is varied over a range of 1-10 to derive the energy variation wrt. the interference variation in the channel. To allocate the available secondary spectrum, the obtained energy ' $E$ ' is used as the allocation parameter. In the data flow process, the optimal encoding mode can be determined by reaching the best trade-off between the amount of coding bits and the obtained service quality. This problem can be modelled as a maximum sum rate optimization problem [1]. This MSR optimization is developed to achieve the objective of fairness of resource utilization in a heterogeneous network. The MSR optimization is governed for allocation of the maximum rate of data flow achieving maximum spectral efficiency. To achieve better service quality for the spectrum allocation, a distribution optimization is proposed. To govern 
quality, a cost function with distortion and rate allocation is defined, subjected to minimization of distortion (D), subjected to an allocation free secondary spectrum ' $R$ ' constraint to the allocation rate $R_{c}$, which is given by $\gamma_{a v 1}^{t}$. The optimization operation of the spectrum is defined as

$$
\min _{\{m\}}\{D\} \text { subject to } R \leq R_{c}
$$

which indicates that the encoder should satisfy the minimum distortion 'D.'

The allocation of spectrum to a user with the data rate satisfying the instantaneous fairness utility function $\theta^{t}$ util was developed [1]. However, in real-time data traffic, such as video streaming or VoIP services, the fairness is defined by the offered BER, controlled by the allocation of data rate. In practical usage, in the network abstraction layer (NAL), the network operates at a different level to provide proper allocation of resources under different data traffic conditions. In wireless communication, the transmission channel is timevarying and distortive in nature. For minimization of error during signal propagation, an independent channel model is used, deriving the bit error rate (BER) of the transmission channel. The packet loss probability ' $\rho$ ' during the transmission of ' $\mathrm{L}$ ' bits for a given BER is defined by

$$
\rho=1-(1-\text { ber })^{L}
$$

The quality utility function $\gamma_{\text {int }}^{t}$ is then defined by

where,

$$
\begin{aligned}
\gamma_{u t i l_{\varphi}}^{t} & =\frac{\gamma_{\varphi}^{t}-\gamma_{\min }^{t}}{\gamma_{\text {max }}^{t}-\gamma_{\min }^{t}} \\
\gamma_{\varphi}^{t} & =\frac{\sum_{u \in U} \gamma_{u \varphi}^{t}}{k} \\
\gamma_{u \varphi}^{t} & =\left(\gamma_{u a}^{t}+L\right)
\end{aligned}
$$

where $\gamma_{u \varphi}^{t}$ is the allocated rate with the primary data rate $\gamma_{u a}^{t}$ and secondary spectrum of L bits. During the transmission process, the packet stream is divided into L-bit slices represented as $s_{n, m}$. For the Tx of the $\mathrm{m}^{\text {th }}$ slice in the $\mathrm{n}^{\text {th }}$ frame, the BER is defined by ber $_{n, m}$, which is the channel BER for the transmission, and $\rho_{n, m}$ is the packet loss rate for a slice $s_{n, m}$. To find the optimal bits of length ' $L$,' minimizing the packet loss $\rho$, the optimization was subjected to $\min (\rho)$ and $\max (\mathrm{L})$.

$$
\min (l) \Rightarrow \max (L)
$$

To generate the data transmitting bits ' $\mathrm{L}$ ' for demand service quality, given by required BER. An optimal value of $L$ is derived, obtaining minimal packet loss. In the operation of rate allocation, the fairness utility function is governed by two levels of maximum or minimum spectral utility functions. The utility component in this case is normalized to two levels, $[0,1]$. While this is developed with the concern of maximum or minimum spectrum utilization, the traffic flow per node is not considered. Data traffic on a heterogeneous node is dynamic in nature, and the two-level normalization process is only for spectrum utilization, wherein this is limited at the device level. The issue arises when the nodes are highly congested due to variable data traffic from different sources, flowing in at different data rates. Though the optimal spectrum utilization is used, if the nodes are blocked at the node level, the network will fail to deliver the demanded service rate. Hence, in addition to spectrum utility function, a device-level optimization is required. To achieve this objective, a rate interference approach is proposed in consideration of the data flow per node for the allocated spectrum. This update in rate allocation results in higher spectrum efficiency in consideration of the cognitive property of the CuE. In addition to spectrum efficiency, a fairness optimization is applied to achieve a higher service level in Heterogeneous Networks.

\section{B. Rate-inference optimization}

While the suggested approach of rate allocation for the fairness approach is observed to be very effective in data flow, when applied to the data flow over a wireless channel with multiple sources, it is observed that the rate of transmission and the offered quality need to be optimized. In the approach of data traffic data flow, due to remote capturing, the allocated resources of power and spectrum will be limited. In such a case, the proper utilization of resources and intermediate node support is highly required to achieve greater performance. The approach of observing for error allocation coding is simpler,and effective for service data flow, but without control of the flow, this allocation may not result in effective service quality at receiving units due to the incurred latency issue. Hence, the access control is also needed with spectrum allocation; with this objective, a buffer-based management scheme to control the rate of allocation was proposed. As in the proposed work, each intermediate node is considered to be a router, and each node encounters multiple data traffic at this node and observes heavy congestion. To overcome this issue, buffer management is applied. The cross-layer optimization of service stream data traffic at the router level was proposed in [14]. The approach of coding was introduced at the network abstraction layer (NAL), where the buffer-based congestion control follows buffer management and the relative quality of service (QoS) is mapped to schedule the rate of data traffic flow. The cross-layer approach processes the measured buffer length and derives the packet En buffer or doping probability based on receiving data traffic. At the Service coding layer, the communication data are blocked into slices and passed to NAL for rate allocation. The method computes the utility function ' $^{\mathrm{t}}$ util ' as

$$
\gamma_{\text {util }}^{\mathrm{t}}=\left\{\begin{array}{c}
0 ; \quad \theta_{\text {util }}^{t}<\gamma_{\text {min }}^{\mathrm{t}} \\
1 ; \quad \theta_{\text {util }}^{t}>\gamma_{\text {max }}^{\mathrm{t}} \\
\max _{p} \times \frac{\gamma^{\mathrm{t}}{ }_{\text {util }}-\gamma_{\text {min }}^{\mathrm{t}}}{\gamma_{\text {max }}^{\mathrm{t}}-\gamma_{\text {min }}^{\mathrm{t}}} ; \text { otherwise }
\end{array}\right.
$$

where $\max _{p}$ is defined as the maximum number of packets exchanged over a node. In the approach of cross-layer modelling, $\theta_{\text {util }}^{t}$ is updated by $1-\phi^{t}$, where $\phi^{t}$ is the update applied in period $\mathrm{t}$ and $\phi$ is the constant value 1.001 defined as the random exponential marking.

The update factor is occasionally updated according to the average buffer length and the input rate and output rate of the buffer. This approach controls the flow of data traffic by accepting or dropping service packets based on the probability index $\phi^{t}$ and the importance of the packet. The factor is incremented if the input rate exceeds the output rate and decremented otherwise. The approach of traffic flow control at node level is defined by the following algorithm. 


\section{Algorithm: Flow control Rate Allocation}

Calculate the average buffer length $q_{1}(t)$ in period $t$;

Receive packeto;

Calculate the utility function $\gamma^{\mathrm{t}}{ }_{\text {util }}$;

Randomize a number $\mu$;

if $\left(\gamma^{\mathrm{t}}{ }_{\text {util }}<\mu\right)$

En buffer packet $\sigma$;

else

re-traffic the packet $\sigma$ with $U=\arg _{i \in[I, L]} \min U(i)$;

buffer packet $\sigma$;

\section{End if}

Where,

$U_{i}(t)$ The important index of the $i$ packet in the buffer in period $t$

The rate allocation is defined by,

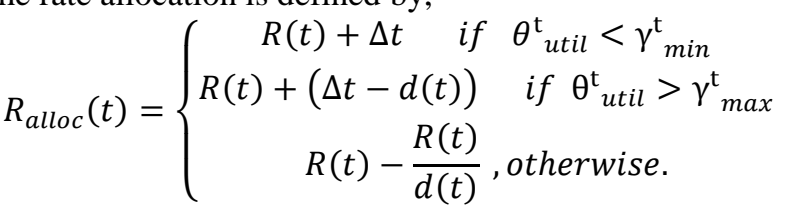

where

$R_{\text {alloc }}(t)=$ allocated data rate

$R(t)=$ full rate

$\Delta t=$ incremental data rate

$\gamma_{\text {min }}^{\mathrm{t}}=$ minimum limit of the buffer

$\gamma_{\text {max }}^{\mathrm{t}}=$ maximum limit of the buffer

In the above equation, the allocated data rate is varying with respect to the node congestion level. If the current buffer length is at the minimum level, the data will be allocated with an increment of ' $\Delta t$.' If the current buffer length in a node is in between the minimum and maximum utility level, the allocated data rate is governed by the traffic flow. Similarly, if the current buffer length exceeds the maximum limit, which represents a traffic flow, the allocated data rate will vary according to the offered traffic utility function $\theta_{u t i l}^{t}$.

\section{EXPERIMENTAL RESULTS}

To evaluate the operational performance of the proposed approach, a MATLAB simulation model is developed, where a distributed wireless Heterogeneous network is simulated with the proposed optimization attribute of the rate flow control approach and compared with the conventional approach of multi-attribute optimization [1] and QoS-based buffer management [14]. For the simulation, a network is defined in Table I with the following network parameters:

For the analysis of the proposed rate flow attribute-based coding approach over the conventional multi-attribute control approach, different networks with varying node distributions are used. Node densities of 30 and 40 terminals are simulated, and the obtained observations are illustrated below.

With the network under consideration, a network with randomly distributed terminals is modelled. The randomly distributed network architecture used for the simulation is illustrated in Fig. 3. The terminals are defined in a random manner with the topology of $\mathrm{N}$ terminals scattered at randomly distributed $\mathrm{X}$ and $\mathrm{Y}$ coordinates. The terminals are defined with random resources of node powers and available spectrum.

The coverage region for each node is illustrated in Fig. 4. The bounded region for each node is computed by the possible range coverage of each node. The communication range of each terminal is defined by its protocol defined for each node, and the terminal in this coverage range is used for the computation of an access link possibility.

TABLE I

Simulation NeTWORK PARAMETERS

\begin{tabular}{ll}
\hline \hline Network parameter & Values \\
\hline \hline Node distribution in the & Random \\
network & Random \\
Power allocation & IEEE 802.11, \\
Communication protocol & LTE $(4 G)$ \\
& $200 \times 200$ \\
Network area & $1-5$ \\
Number of communication & \\
terminals & $3 M$ \\
Memory size / node $(M)$ & $0.15 \times M$ \\
$\gamma_{\min }$ & $0.75 \times M$ \\
$\gamma_{\text {max }}$ & 0.1 \\
\hline Initial blockage probability & \\
\hline
\end{tabular}

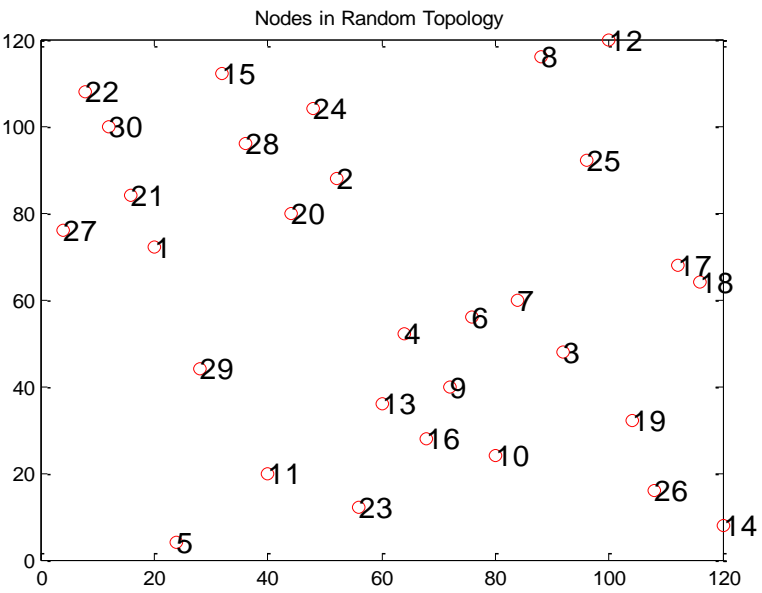

Fig. 3. Randomly distributed access points for a terminal density of 30

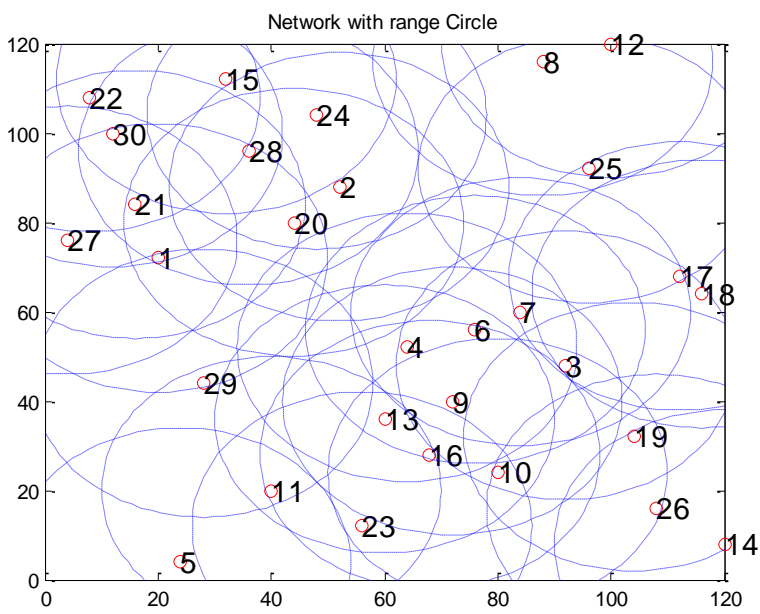

Fig. 4. Network with coverage regions marked 


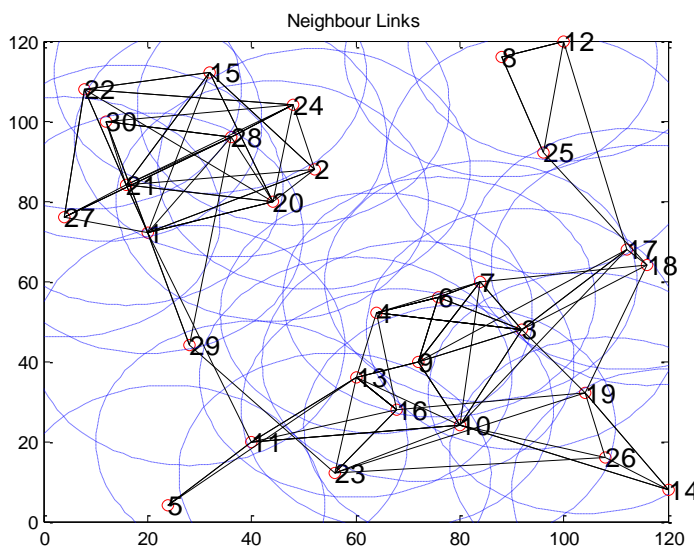

Fig. 5. Network with 1-Hop neighbours

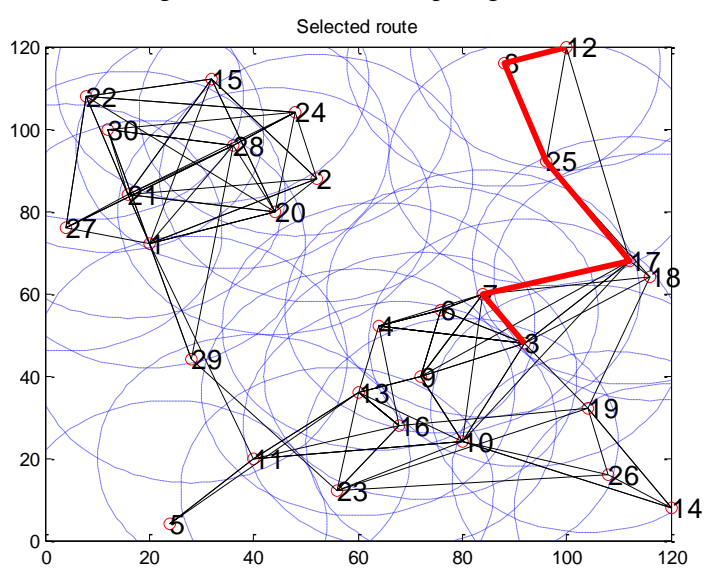

Fig. 6. Network with a selected path for communication

The network with 1-hop neighbours is illustrated in Fig. 5. Each node computes the distance of nodes with all other terminals, and the distance below the communication range is defined as a 1-hop neighbour for the refereeing node. In the process of neighbour discovery, the Terminal distances falling below the communication range are declared as direct neighbours, and each node exchanges their one-hop neighbour details to formulate a link network.

With the one-hop neighbour list, an AODV routing protocol is developed to evaluate the route from a given source to the destination. The routes are generated via the data exchange from source to destination in a broadcasting manner. A forward and backward tracing method is applied to obtain the route parameters used for forwarding packets. Among the generated routes, a path with minimum hops (i.e., the shortest path) is selected for communication. The selected path for the developed network is as illustrated in Fig. 6.

In the process of communication, data are forwarded along the path selected from source to destination via intermediate terminals. During the process of data exchange, the packets are buffered, and based on the computed congestion level, packets are forwarded. To transfer the packets, the per node forwarding data rate is computed based on the congestion level, and allocated data rate packets are released or queued in the node. The queued packets internally build an overhead in the network. This overhead is defined as the number of packets queued for processing at each node. With the forwarding of packets from source to destination at each intermediate node, packets are buffered; the overhead observed is hence presented in Fig. 7.

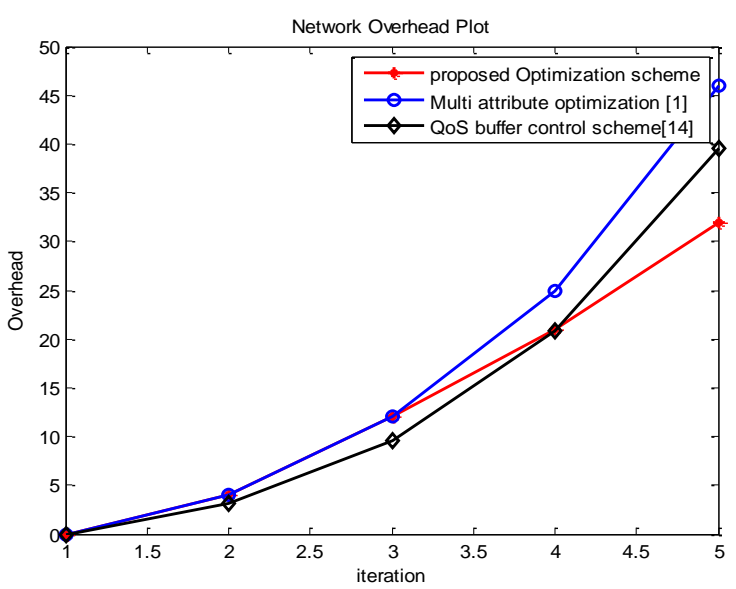

Fig. 7. Network overhead with packet forwarding

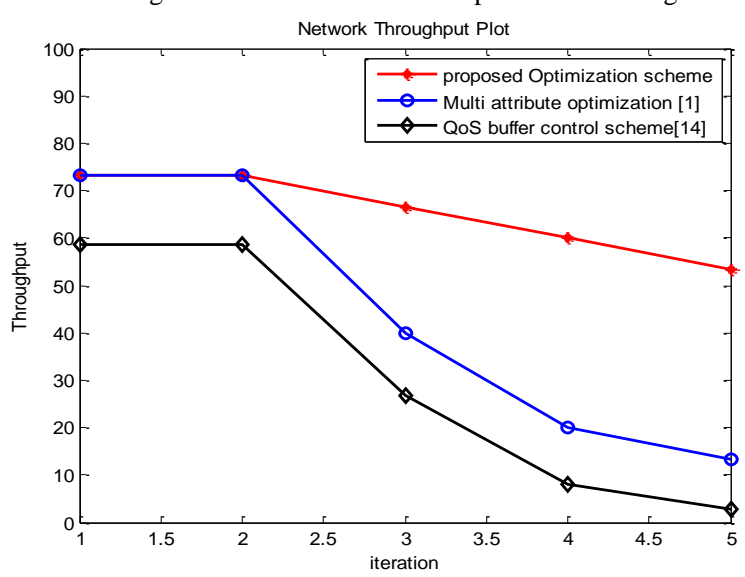

Fig. 8. Network throughput with the forwarding of packets

Due to the rate flow attribute coding, the congestion is controlled over a limit of buffering in contrast to buffering to a higher level of buffer queue. Due to early control and rate flow attribute estimation, the overhead for such a network is observed to be minimized with the forwarding of packets.

The network throughput for the developed network is illustrated in Fig. 8. Due to the higher data rate of packet forwarding in the proposed approach, it is observed that more packets are received, hence resulting in higher throughout in the network. The throughput of these simulated systems is defined as the number of packets generated over packets received for an observed communication time period. The throughput is observed to be the same for the first 1-hop forwarding, and in such a case, the buffering is observed to be minimized; hence, the allocated data rate is improved. However, as the amount of packet forwarding increases, the congestion level increases, resulting in the decrement of throughput, which is maintained to be higher and linear in the case of rate flow attribute coding.

The observed end-to-end delay factor for the developed system is presented in Fig. 9. The delay for the rate control approach is higher than that of the rate flow attribute coding, as the buffering of data at each node is minimized at the node level and the packets are released faster. The congestion level in such coding is developed in a rate flow attribute manner, wherein packets are buffered in the queue based on the rate control approach, which builds the forwarding delay for each node.

The buffered Q length for such a system is presented in Fig. 10. The $Q$ lengths are measured as the volumes of data packets buffered with an increase in forwarding of data packets. It is 
observed that the Q length of buffering is reduced for rate flow attribute coding due to the increment in the data rate. The queuing is, however, observed to be equal in the initial communication phase and gradually increases with the forwarding of packets.

The allocated data rate for the developed method over the simulation network is as outlined in Fig. 11. The terminal is communicated for the given data packet, and each level of buffering of these terminals computes the allocable data rate for forwarding. This forwarding results in proper control of congestion and hence results in efficient network performance.

To evaluate the impact of node density, a similar observation is made for the node density of 40 terminals. The obtained observation for such a network is illustrated in the following observation.

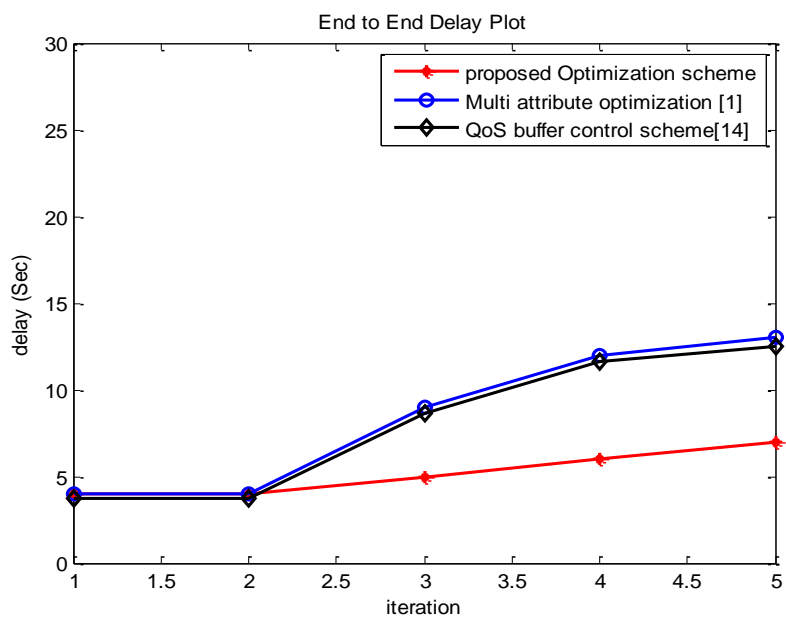

Fig. 9. Observed delay for the simulated network with a node density of 30

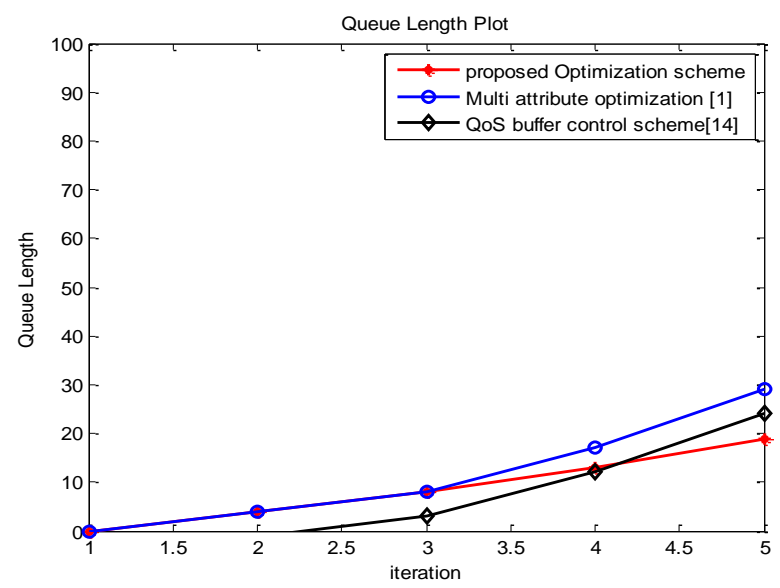

Fig. 10. Buffered Q_length with an increase in forwarded packets

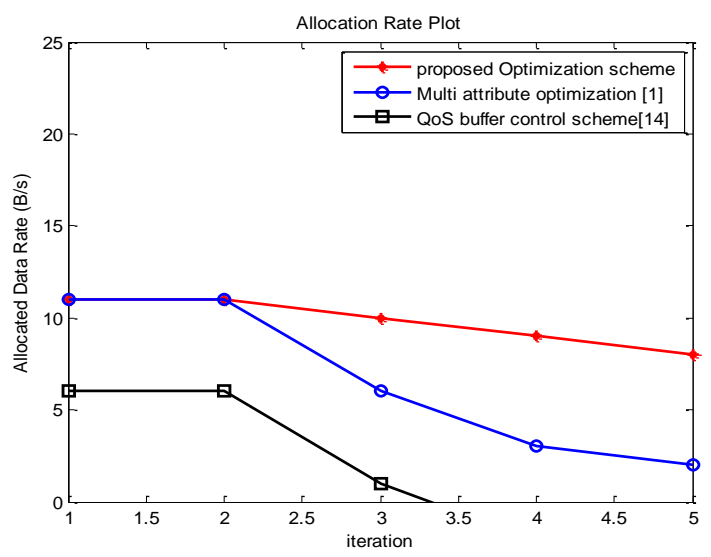

Fig. 11. Allocated data rate with forwarding of packet

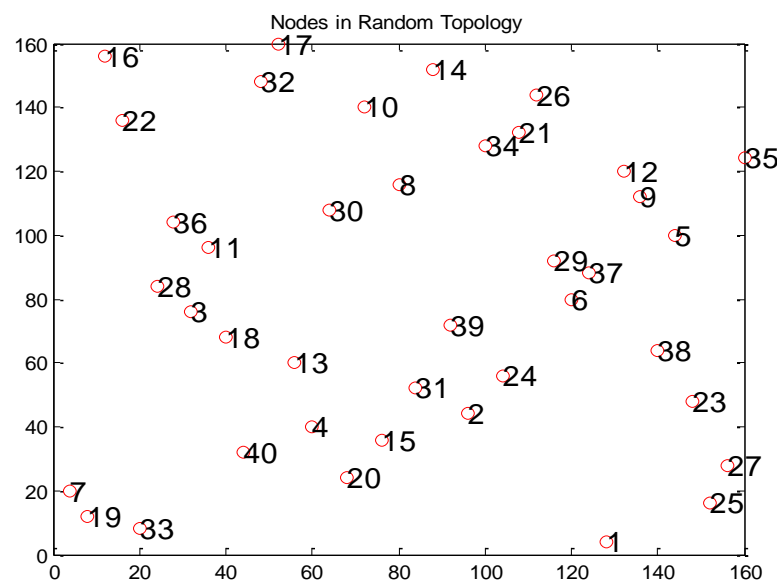

Fig. 12. Network topology for randomly distributed terminals with a node density of 40

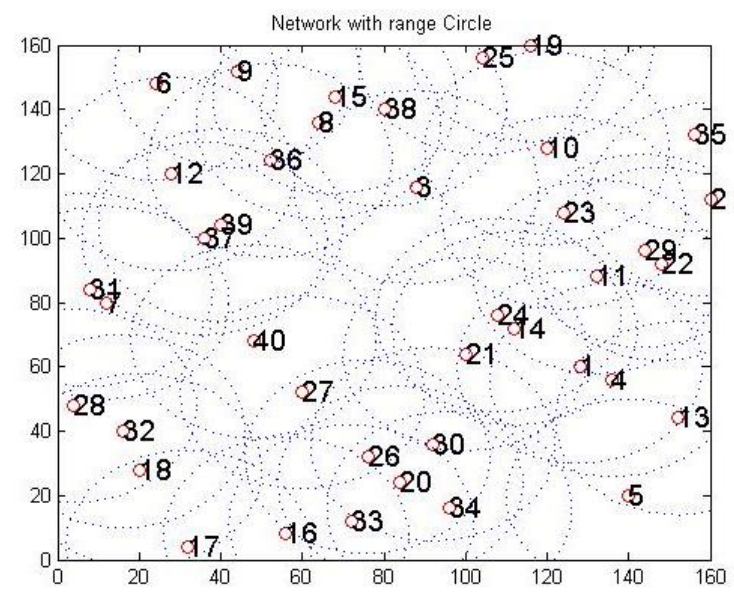

Fig. 13. Marked coverage regions for each node in the network 


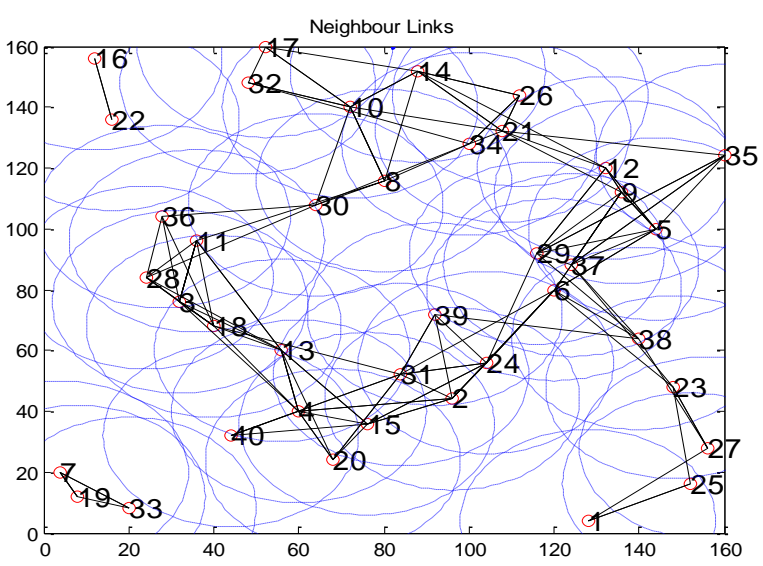

Fig. 14. Network with 1-hop neighbour links

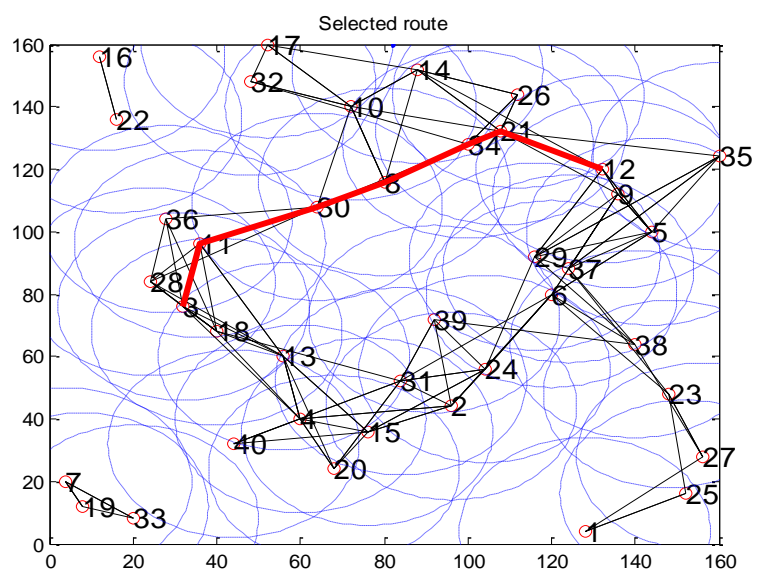

Fig. 15. Network with a selected route for communication

The node distribution, coverage range, neighbour links and selected path for communication are as illustrated in Figures 12-15, respectively.

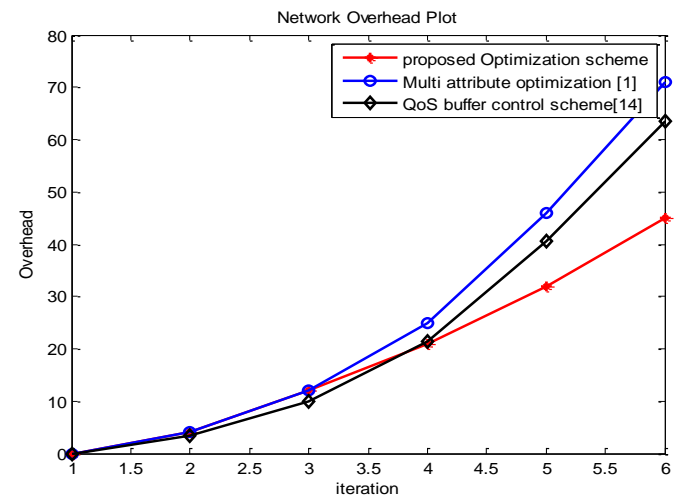

Fig. 16. Network overhead with an increase in the forwarding of packets

The network overhead in such a case is observed to be reduced from 122 to 71 . The overhead is minimized due to the existence of more terminals to exchange the data, which results in faster data exchange. However, due to the non-optimal rate control approach, the overhead is observed to be higher in the rate control approach in comparison to the rate flow attribute approach.

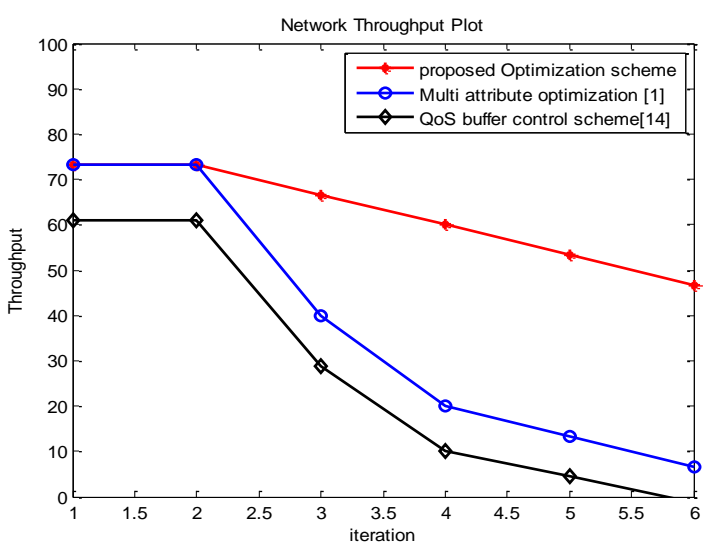

Fig. 17. Network throughput for the developed network for a node density of 40

The network throughput for this developed network is observed to be improved from 30 to 52 in the case of the rate flow attribute-based control approach. This is observed to be minimized in the case of the rate control method due to the higher data buffering per node.

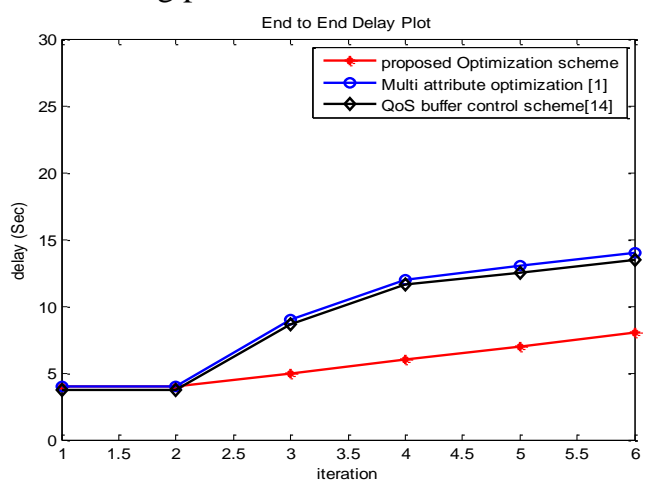

Fig. 18. End-to-end delay for the developed network

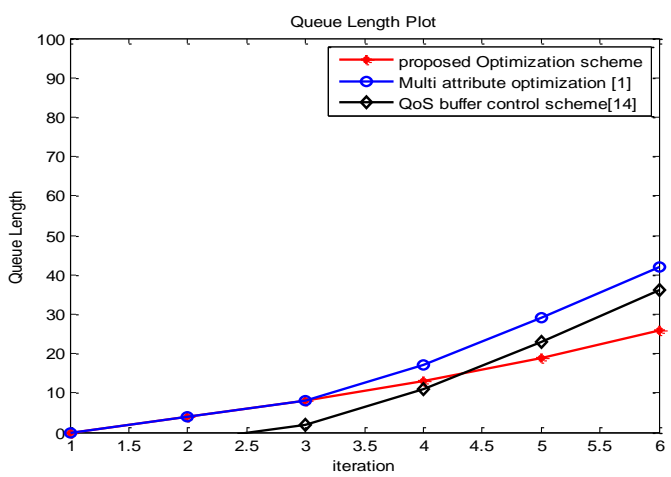

Fig. 19. Observed Q-Length at the terminal for the developed network

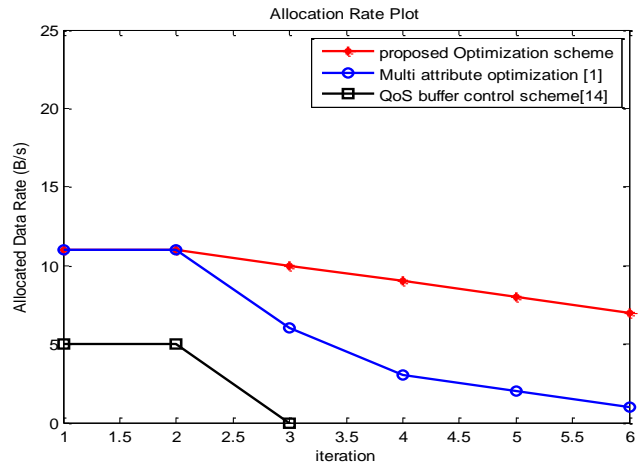

Fig. 20. Allocation of data rate for the developed methods 
The observed delay factor is minimized from 10 to 7 for higher node density, the Q_length is decreased from 42 to 25 , and the allocable data rate is increased from 5 to 7 for the developed rate flow attribute method. The obtained observations for the delay, Q_length and allocated data rate is presented in Figures 18-20. The observation made for a node density of 40 terminals is presented in Table II. The allocated data rate could be observed to be improvised in rate flow attribute-based coding as presented in Table II. The average queue length is hence reduced from 29 to 19 in this case. The network throughput is observed to be increased from 13.34 to 53.36 in such case. The delay is minimized from 13 to 7 for the rate flow attribute-based control approach in comparison to the rate control approach.

TABLE II

Comparative Observation of the Three DevelOped Methods at A NODE DENSITY OF 40

\begin{tabular}{|c|c|c|c|c|}
\hline Method & Allocated DR & $\begin{array}{c}\text { Average } \\
\text { Q_Length }\end{array}$ & Throughput & Delay \\
\hline $\begin{array}{c}\text { Multi- } \\
\text { attribute } \\
\text { optimization } \\
{[1]}\end{array}$ & {$\left[\begin{array}{lllll}1 & 1 & 11 & 6 & 3\end{array}\right]$} & {$\left[\begin{array}{lllll}0 & 4 & 8 & 17 & 29\end{array}\right]$} & $\begin{array}{l}{\left[\begin{array}{llll}73 & 73 & 40 & 20 \\
13]\end{array}\right.}\end{array}$ & $\begin{array}{l}{\left[\begin{array}{lll}4 & 4 & 9 \\
12 & 13\end{array}\right]}\end{array}$ \\
\hline $\begin{array}{c}\text { QoS } \\
\text { Buffer } \\
\text { optimization } \\
{[14]}\end{array}$ & {$\left[\begin{array}{lllll}11 & 11 & 8 & 5 & 5\end{array}\right]$} & {$\left[\begin{array}{lllll}0 & 4 & 8 & 15 & 22\end{array}\right]$} & $\begin{array}{l}{\left[\begin{array}{llll}73 & 73 & 4732 \\
27\end{array}\right]}\end{array}$ & $\begin{array}{l}{\left[\begin{array}{lll}4 & 4 & 7 \\
10 & 11\end{array}\right]}\end{array}$ \\
\hline $\begin{array}{c}\text { Proposed } \\
\text { approach } \\
\text { optimization }\end{array}$ & {$\left[\begin{array}{lllll}11 & 11 & 10 & 9 & 8\end{array}\right]$} & {$\left[\begin{array}{lllll}0 & 4 & 8 & 13 & 19\end{array}\right]$} & $\begin{array}{l}{\left[\begin{array}{lll}73.33 & 73.33 \\
66 & 60 & 53.6\end{array}\right]}\end{array}$ & $\begin{array}{l}{[445} \\
67]\end{array}$ \\
\hline
\end{tabular}

\section{CONCLUSION}

In the suggested approach, the cognitive property of devices is utilised for a secondary spectrum sensing operation to achieve a higher data rate allocation under ideal spectrum availability. In the flow control approach, the buffer control mechanism is improved for the given quality of service, where the traffic monitoring is introduced based on the allocated data rate and buffer status. An improvement in the network operation metric of throughput is observed.

\section{REFERENCES}

[1] R. Amin, J. Martin, J. Deaton, L. A. DaSilva, A. Hussien, A. Eltawil, "Balancing spectral efficiency, energy consumption, and fairness in future heterogeneous wireless systems with reconfigurable devices," IEEE Journal on Selected Areas in Communications, vol. 31(5), pp. 969-980, 2013. DOI: 10.1109/JSAC.2013.130515

[2] S. K. Sharma, S. Chatzinotas, B. Ottersten. "Interference alignment for spectral coexistence of heterogeneous networks," EURASIP Journal on Wireless Communications and Networking , vol. 201(1), pp. 1-14, 2013. DOI: 10.1186/1687-1499-2013-46

[3] S.V. Hanly, C. Liu, P. Whiting, "Capacity and stable scheduling in heterogeneous wireless networks." IEEE Journal on Selected Areas in Communications vol. 33(6), pp. 1266-1279, 2015. DOI: 10.1109/JSAC.2015.2416971

[4] H. M. Tahir, A. H. Al-Ghushami, Z. R. Yahya, "Selection of access network using cost function method in heterogeneous wireless network." Multimedia Computing and Systems (ICMCS), 2014 International Conference on. IEEE, 2014.
[5] A. Khandekar, N. Bhushan, J. Tingfang, V. Vanghi, "LTE-advanced: Heterogeneous networks", In Wireless Conference (EW), 2010 European (pp. 978-982), IEEE, 2010.

[6] W. Mansouri, K. Mnif, F. Zarai, M. S. Obaidat, "A new multi-rat scheduling algorithm for Heterogeneous wireless networks", Journal of Systems and Software, Elsevier, 2015

[7] F. W. Karam, T. Jensen, "On schemes for supporting QoS and mobility in heterogeneous networks." Proceedings of the 7th International Conference on Frontiers of Information Technology. ACM, 2009.

[8] M. Liu, Y. Teng, M. Song. "Tradeoff Analysis between Spectrum Efficiency and Energy Efficiency in Heterogeneous Networks (HetNets) Using Bias Factor." Journal of Communications 10.10, 2015.

[9] K. Son, E. Oh, B. Krishnamachari. "Energy-efficient design of heterogeneous cellular networks from deployment to operation."Computer Networks vol. 78, pp. 95-106, 2015.

[10] G. He, S. Zhang,, Y. Chen, S. Xu, "Spectrum efficiency and energy efficiency tradeoff for heterogeneous wireless networks", In 2013 IEEE Wireless Communications and Networking Conference (WCNC), pp. 2570-2574, IEEE, 2013

[11] R. Q. Hu, Y. Qian, "An energy efficient and spectrum efficient wireless heterogeneous network framework for $5 \mathrm{G}$ systems." IEEE Communications Magazine vol. 52.5 (2014), pp. 94-101, 2014.

[12] J. B. Rao, A. O. Fapojuwo, "Analysis of Spectrum Efficiency and Energy Efficiency of Heterogeneous Wireless Networks With Intra-/Inter-RAT Offloading," IEEE Transactions on Vehicular Technology 64.7 (2015), pp. 3120-3139, 2015.

[13] H. Shi, J. Li, Z. Li, H. Ju. "Dynamic spectrum allocation in heterogeneous wireless networks under interference constraints across the cell coverage." Wireless Communications and Mobile Computing 15, no. 7 (2015), pp. 1188-1197, 2015

[14] B. Iancu, D. Vasile, A. Peculea. "A novel end-to-end QoS framework over heterogeneous networks-an architectural approach."Acta Technica Napocensis 51.2 (2010): 42, 2010.

[15] L. Badia, M. Miozzo, M. Rossi, M. Zorzi, „Routing schemes in heterogeneous wireless networks based on access advertisement and backward utilities for QoS support [Quality of Service based Routing Algorithms for Heterogeneous Networks],“. IEEE Communications Magazine,45(2), pp. 67-73, 2007.

[16] K. Yang, Y. Wu, H. H. Chen. "QoS-aware routing in emerging heterogeneous wireless networks [Quality-of-Service-Based Routing Algorithms for Heterogeneous Networks]." IEEE Communications Magazine 45.2 (2007), pp. 74-80, 2007.

[17] F. Richter, A.. J. Fehske, P. Marsch, G.P. Fettweis, (2010, May). Traffic demand and energy efficiency in heterogeneous cellular mobile radio networks. In Vehicular Technology Conference (VTC 2010-Spring), 2010 IEEE $71^{\text {st }}$, pp. 1-6. IEEE.

[18] G. Fodor, E. Anders, A. Tuoriniemi. "Providing quality of service in always best connected networks." IEEE Communications Magazine 41.7 (2003), pp. 154-163, 2003.

[19] G. Aristomenopoulos, T. Kastrinogiannis, Z. Li, S. Papavassiliou, (2011), "An autonomic qos-centric architecture for integrated heterogeneous wireless networks", Mobile Networks and Applications, 16(4),pp. 490504, 2011.

[20] J. C. Clement, B. B. Reddy, D. S. Emmanuel, "An Energy-Efficient Cooperative Spectrum Sensing Strategy with Robustness Against Noise Uncertainty for Cognitive Radio Networks." Arabian Journal for Science and Engineering (2015), pp. 1-7, 2015.

[21] Z. Dai, J. Liu, C. Wang. "QoS-based device-to-device communication schemes in heterogeneous wireless networks." IET Communications 9.3 (2015), pp. 335-341, 2015

[22] R. Xie, F. R. Yu, H. Ji. "Dynamic resource allocation for heterogeneous services in cognitive radio networks with imperfect channel sensing." IEEE Transactions on Vehicular Technology 61.2 (2012): 770780,2012

[23] W. Sheikh, A. Ghafoor, "Quality-of-service routing in heterogeneous networks with optimal buffer and bandwidth allocation", In2007 IEEE International Conference on Communications (pp. 480-485) IEEE, 2007 